\title{
Significance of NT-pro-BNP in acute exacerbation of COPD patients without underlying left ventricular dysfunction
}

This article was published in the following Dove Press journal:

International Journal of COPD

13 April 2017

Number of times this article has been viewed

\author{
Muhammad Adrish' \\ Varalaxmi Bhavani Nannaka ${ }^{2}$ \\ Edison J Cano ${ }^{3}$ \\ Bharat Bajantri' \\ Gilda Diaz-Fuentes' \\ 'Division of Pulmonary and Critical \\ Care Medicine, Bronx-Lebanon \\ Hospital Center, Icahn School of \\ Medicine at Mount Sinai, ${ }^{2}$ Department \\ of Critical Care Medicine, Montefiore \\ Medical Center, The University \\ Hospital for Albert Einstein College \\ of Medicine, ${ }^{3}$ Department of Medicine, \\ Bronx-Lebanon Hospital Center, Icahn \\ School of Medicine at Mount Sinai, \\ Bronx, NY, USA
}

Background: B-type natriuretic peptide (BNP) and the N-terminal fragment of pro-BNP (NT-pro-BNP) are established biomarkers of heart failure. Increased levels of natriuretic peptide (NP) have been associated with poor outcomes in acute exacerbation of COPD (AECOPD); however, most studies did not address the conditions that can also increase NT-pro-BNP levels. We aimed to determine if NT-pro-BNP levels correlate with outcomes of AECOPD in patients without heart failure and other conditions that can affect NT-pro-BNP levels.

Methods: We conducted a retrospective study in patients hospitalized for AECOPD with available NT-pro-BNP levels and normal left ventricular ejection fraction. We compared patients with normal and elevated NT-pro-BNP levels and analyzed the clinical and outcome data.

Results: A total of 167 of 1,420 (11.7\%) patients met the study criteria. A total of $77 \%$ of male patients and $53 \%$ of female patients had elevated NT-pro-BNP levels ( $P=0.0031)$. NT-pro-BNP levels were not associated with COPD severity and comorbid illnesses. Log-transformed NTpro-BNP levels were positively associated with echocardiographically estimated right ventricular systolic pressure $(r=0.3658 ; 95 \%$ confidence interval [CI]: $0.2060-0.5067 ; P<0.0001)$. Patients with elevated NT-pro-BNP levels were more likely to require intensive care (63\% vs $43 \%$; $P=0.0207)$ and had a longer hospital length of stay $(P=0.0052)$. There were no differences in the need for noninvasive positive pressure ventilation $(P=0.1245)$ or mechanical ventilation $(P=0.9824)$ or in regard to in-hospital mortality $(P=0.5273)$.

Conclusion: Patients with AECOPD and elevated NT-pro-BNP levels had increased hospital length of stay and need for intensive care. Based on our study, serum NT-pro-BNP levels cannot be used as a biomarker for increased mortality or requirement for invasive or noninvasive ventilation in this group of patients.

Keywords: acute exacerbation of COPD, NT-pro-BNP, natriuretic peptide, COPD

\section{Background}

COPD is a leading cause of morbidity and mortality worldwide and is associated with a significant economic burden. In 2014, 15.7 million Americans reported that they were diagnosed with COPD. ${ }^{1}$ Prevalence of COPD increases with age, ranging from $2.6 \%$ in adults aged $18-34$ years of age to $12.3 \%$ in adults aged 75 years or older. ${ }^{1}$

Studies show that $>50 \%$ of adults with low pulmonary function do not know that they have COPD, suggesting that actual estimates could be higher. ${ }^{2}$ COPD not only leads to limitations in daily activities but also leads to increased economic burden. Work force participation estimates suggest a significant decline in adults with COPD as well as limitations in the type and amount of work that they can perform with restricted activity days ranging from 27 to 63 days per year compared with adults without COPD. ${ }^{3}$
Correspondence: Muhammad Adrish Division of Pulmonary and Critical Care Medicine, Bronx-Lebanon Hospital Center, Icahn School of Medicine at Mount Sinai, 1650 Selwyn Avenue, Suite I2F, Bronx, NY 10457, USA

Tel + I 7| 8960 I234

Email aadrish@hotmail.com 
B-type natriuretic peptide (BNP) is derived from the prohormone pro-BNP, an intracellular, 108-amino acid precursor protein, which is cleaved into two fragments and released by myocytes, yielding BNP and the 76-amino acid N-terminal fragment of pro-BNP (NT-pro-BNP).

BNP and NT-pro-BNP are established biomarkers of heart failure $(\mathrm{HF})^{4}$ and are primarily used for diagnosis, risk stratification, and management. Elevated natriuretic peptide levels have also been observed in patients with COPD without HF, likely originating from the left and right sides of the heart. Cor pulmonale, secondary pulmonary hypertension, and hypoxemia represent important stimuli for the release of natriuretic peptides from the right side of the heart and increase BNP gene expression..$^{5-7}$

Several studies have suggested the utility of BNP in predicting the outcomes of COPD. A prospective, single-center study $^{8}$ of stable patients with COPD in the ambulatory setting suggested that NT-pro-BNP predicts survival in unadjusted survival plots; however, the association was not significant when adjusted for the presence of a dilated left atrium, aortic stenosis, and left ventricular systolic dysfunction. Another study of patients with AECOPD suggested an association between elevated NT-pro-BNP levels and 30-day mortality. ${ }^{9}$ That study was limited to include only those patients with known cardiovascular diseases. Hoiseth et $\mathrm{al}^{10}$ showed an association between NT-pro-BNP and long-term mortality in patients with AECOPD; however, $\sim 12 \%$ of the patients in that study did not have spirometrically confirmed COPD. A metaanalysis by Buchan et $\mathrm{al}^{11}$ found that most of the studies correlating COPD with NT-pro-BNP levels included left ventricular dysfunction as a primary or secondary end point.

We aimed to assess the correlation between NT-pro-BNP levels and outcomes in patients admitted to the hospital with AECOPD while accounting for known confounders of NT-pro-BNP elevation. The primary outcomes included the needs for noninvasive positive pressure ventilation (NIPPV), mechanical ventilation (MV), tracheostomy, and admission to the intensive care unit (ICU). In addition, we evaluated mortality and the length of stay (LOS) parameters.

\section{Methods}

This was a retrospective study of all adult patients admitted with AECOPD to our institution from January 2010 to December 2014. We obtained data regarding baseline demographics, Global Initiative for Chronic Obstructive Lung Disease (GOLD) staging, and home oxygen requirement. We reviewed laboratory parameters and chest X-rays at the time of admission, serum NT-pro-BNP levels, and echocardiograms.
The inclusion criteria were available spirometry with post-bronchodilator forced expiratory volume in 1 second $\left(\mathrm{FEV}_{1}\right) /$ forced vital capacity $(\mathrm{FVC})<0.70$, available serum NT-pro-BNP levels at the time of admission, normal left ventricular ejection fraction confirmed by echocardiogram within 12 months of admission, and a clinical diagnosis of COPD exacerbation. We excluded patients with any of the following characteristics: advanced renal disease, systolic HF (ejection fraction $<50 \%$ ), valvular heart disease, acute myocardial infarction, and grade 3 or grade 4 diastolic dysfunction (DD). We included patients with grades 1 and $2 \mathrm{DD}$ in our analysis to evaluate if any correlation with NTpro-BNP exists.

A total of 1,420 patients were admitted with the diagnosis of AECOPD during the study period. Of these, 167 patients $(11.7 \%)$ met our study criteria described earlier and were analyzed.

\section{Statistical analysis}

We compared the clinical characteristics between patients with normal and elevated age-specific NT-pro-BNP levels by $\chi^{2}$ tests for categorical data, Student's $t$-tests for continuous parametric data, and Wilcoxon-Mann-Whitney tests for continuous nonparametric data. Kruskal-Wallis test was used to compare the NT-pro-BNP levels among patients categorized by GOLD stage and ethnicity. We used log-transformed NT-pro-BNP levels to correlate right ventricular systolic pressure (RVSP) and other laboratory results by Spearman correlation tests.

We conducted a multivariable logistic regression analysis of binary outcomes, including NIPPV use, need for intubation and mechanical intubation, and need for new tracheostomy, which were our primary outcomes under focus. A multivariable linear regression analysis was conducted for NT-pro-BNP levels, laboratory data, LOS in the hospital and in the ICU, and the number of days of MV and NIPPV.

We expressed the results as counts (percentages) for discrete variables and as mean values $( \pm$ standard deviation [SD]) for continuous variables. Statistically significant associations were retested using multivariable models to account for interactions and confounders. The $P$-values $\leq 0.05$ were considered statistically significant, and all confidence intervals (CIs) were reported to four decimal places. We employed RStudio V0.98.1103 (RStudio Inc., Boston, MA, USA) and Excel 2010 (Microsoft Corp, Redmond, WA, USA) for the data analyses. This study was reviewed and approved by the institutional review board at Bronx-Lebanon Hospital Center. The study number is 01081503 , which was approved 
on January 8, 2015. The institutional review board waived the need for informed consent for this study due to its retrospective nature. The study followed strict policies to protect patient data confidentiality.

\section{Results}

A total of 167 patients met the study criteria during this period of which 104 (62\%) had elevated age-specific NT-pro-BNP levels. Table 1 shows the general characteristics of the two

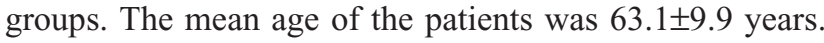
A total of $39 \%$ of the patients were males. There were no differences among ethnic groups in the proportion of patients with elevated or normal NT-pro-BNP levels $(P=0.3624)$ or in the severity of COPD as indicated by GOLD staging or home oxygen requirement. Patients with normal NT-proBNP levels had higher BMI than those with elevated NTpro-BNP levels, but there were no differences between the two groups in terms of underlying comorbid conditions. The median interval between the echocardiogram and hospital admission was 28 days (range: 1-364 days).

Table 2 shows the laboratory values during admission. Patients with elevated NT-pro-BNP levels had lower serum albumin $(P=0.0042)$, higher serum blood urea nitrogen (BUN) levels $(P=0.0164)$, and higher partial pressure of carbon dioxide $(P=0.043)$. There were no differences in serum creatinine, serum bicarbonate, serum troponins, serum $\mathrm{pH}$, and partial pressure of oxygen between the two groups. We also noted that patients with elevated NT-pro-BNP levels had higher RVSPs compared to patients with normal NTpro-BNP levels $(P=0.0002)$. Patients with normal NT-proBNP levels were more likely to have grade 1 or grade $2 \mathrm{DD}$ (87\%) compared with patients with elevated NT-pro-BNP levels $(P=0.0033)$.

In the Pearson correlation analysis (Table 3), there was a significant, moderately positive association between the log-transformed NT-pro-BNP levels and echocardiographically estimated RVSP ( $r=0.3658$; 95\% CI: $0.2060-0.5067$; $P<0.0001$; Figure 1). There were weakly positive relationships between the log-transformed NT-pro-BNP levels and $\mathrm{pCO}_{2}$ levels $(r=0.2113$; 95\% CI: $0.0615-0.3519$; $P=0.0061)$ and serum bicarbonate levels $(r=0.1893 ; 95 \%$ CI: 0.0386-0.3317; $P=0.0143)$.

Clinical interventions and patient outcomes are summarized in Table 4. Patients with elevated NT-pro-BNP levels were more likely to be admitted to the ICU (63\% vs $43 \%$; $P=0.0207$ ), which was evident in the univariate regression model $(\mathrm{OR}=2.22 ; 95 \% \mathrm{CI}: 1.1799-4.2373 ; P=0.0141)$, however, in the multivariable model, it did not reach

Table I Demographic characteristics of patients with AECOPD according to age-specific NT-pro-BNP levels

\begin{tabular}{|c|c|c|c|c|}
\hline Variables & $\begin{array}{l}\text { All cases } \\
(n=167)\end{array}$ & $\begin{array}{l}\text { Normal NT-pro- } \\
\text { BNP }(n=63)\end{array}$ & $\begin{array}{l}\text { High NT-pro- } \\
\text { BNP }(n=104)\end{array}$ & Significance \\
\hline \multicolumn{5}{|l|}{ Demographics } \\
\hline Mean age (SD) & $63.1(9.9)$ & $63.7(9.6)$ & $62.8(10.0)$ & $P=0.5303$ \\
\hline \multicolumn{5}{|l|}{ Sex, n (\%) } \\
\hline Male & $65(39)$ & $15(24)$ & $50(48)$ & $P=0.0031$ \\
\hline Female & $102(61)$ & $48(76)$ & $54(52)$ & \\
\hline Mean BMI (SD) & $29.6(11.9)$ & $32.3(12.8)$ & $27.9(10.9)$ & $P=0.0149$ \\
\hline \multicolumn{5}{|l|}{ Ethnicity, n (\%) } \\
\hline African-American & $91(54)$ & $36(57)$ & $55(53)$ & $P=0.3624$ \\
\hline Hispanic & $67(40)$ & $22(35)$ & $45(43)$ & \\
\hline Others (Asian or White) & $9(5)$ & $5(8)$ & $4(4)$ & \\
\hline \multicolumn{5}{|l|}{ COPD severity, n (\%) } \\
\hline GOLD I & $14(8)$ & $6(10)$ & $8(8)$ & $P=0.8998$ \\
\hline GOLD 2 & $47(28)$ & $20(32)$ & $27(26)$ & $P=0.5299$ \\
\hline GOLD 3 & $76(46)$ & $27(43)$ & $49(47)$ & $P=0.7074$ \\
\hline GOLD 4 & $30(18)$ & $10(16)$ & $20(19)$ & $P=0.7379$ \\
\hline Home oxygen dependant, n (\%) & $65(39)$ & $20(32)$ & $45(43)$ & $P=0.1880$ \\
\hline \multicolumn{5}{|l|}{ Comorbidities, n (\%) } \\
\hline Pulmonary embolism & $5(3)$ & $2(3)$ & $3(3)$ & $P=1.0000$ \\
\hline Atrial fibrillation & $15(9)$ & $3(5)$ & $12(12)$ & $P=0.228 \mathrm{I}$ \\
\hline Renal failure & $12(7)$ & I (2) & II (II) & $P=0.0613$ \\
\hline Liver failure & $2(1)$ & $0(0)$ & $2(2)$ & $P=0.7088$ \\
\hline Malnutrition & $18(11)$ & $6(10)$ & $12(12)$ & $P=0.88 \mathrm{II}$ \\
\hline
\end{tabular}

Abbreviations: AECOPD, acute exacerbation of COPD; BMI, body mass index; BNP, B-type natriuretic peptide; NT-pro-BNP, N-terminal fragment of pro-BNP; $\mathrm{O}_{2}$, oxygen; $\mathrm{SD}$, standard deviation. 
Table 2 Laboratory and imaging results according to age-specific NT-pro-BNP levels in patients with AECOPD

\begin{tabular}{|c|c|c|c|c|}
\hline Variables & $\begin{array}{l}\text { All cases } \\
(n=167)\end{array}$ & $\begin{array}{l}\text { Normal NT-pro- } \\
\text { BNP }(n=63)\end{array}$ & $\begin{array}{l}\text { High NT-pro- } \\
\text { BNP }(n=104)\end{array}$ & Significance \\
\hline \multicolumn{5}{|l|}{ Laboratory } \\
\hline NT-pro-BNP (pg/mL), mean (SD) & 704.I $(1,430.2)$ & $88.3(53.3)$ & $\mathrm{I}, 077.2(\mathrm{I}, 707 . \mathrm{I})$ & $P<0.0001$ \\
\hline Albumin, serum (g/dL), mean (SD) & $3.9(0.4)$ & $4.0(0.4)$ & $3.8(0.4)$ & $P=0.0042$ \\
\hline BUN, serum (mg/dL), mean (SD) & $17.4(9.5)$ & I $4.8(6.2)$ & $18.9(10.7)$ & $P=0.0164$ \\
\hline Creatinine, serum (mg/dL), mean (SD) & $0.9(0.6)$ & $0.8(0.4)$ & $\mathrm{I} .0(0.7)$ & $P=0.056 \mathrm{I}$ \\
\hline Troponin elevation, n (\%) & $6(4)$ & $0(0)$ & $6(6)$ & $P=0.154 \mid$ \\
\hline $\mathrm{pH}$, mean $(\mathrm{SD})$ & $7.3(0.1)$ & $7.3(0.1)$ & $7.3(0.1)$ & $P=0.3222$ \\
\hline $\mathrm{PCO}_{2}(\mathrm{mmHg})$, mean $(\mathrm{SD})$ & $60.4(21.7)$ & $56.0(14.5)$ & $63.0(24.6)$ & $P=0.043$ \\
\hline $\mathrm{pO}_{2}(\mathrm{mmHg})$, mean $(\mathrm{SD})$ & $74.4(38.8)$ & $70.7(34.8)$ & $76.7(40.8)$ & $P=0.2644$ \\
\hline Bicarbonate, serum (mEq/L), mean (SD) & $28.3(5.0)$ & $27.4(3.8)$ & $28.8(5.6)$ & $P=0.1988$ \\
\hline \multicolumn{5}{|l|}{ Echocardiogram } \\
\hline RVSP (mmHg), mean (SD) & $42.2(12.8)$ & $37.5(9.2)$ & $45.5(14.0)$ & $P=0.0002$ \\
\hline \multicolumn{5}{|l|}{$\mathrm{DD}, \mathrm{n}(\%)$} \\
\hline No DD & $44(26)$ & $8(13)$ & $36(35)$ & $P=0.0073$ \\
\hline Grades I and 2 DD & $123(74)$ & $45(87)$ & $54(65)$ & \\
\hline
\end{tabular}

Abbreviations: AECOPD, acute exacerbation of COPD; BNP, B-type natriuretic peptide; BUN, blood urea nitrogen; DD, diastolic dysfunction; NT-pro-BNP, N-terminal fragment of pro-BNP; $\mathrm{PCO}_{2}$, partial pressure of carbon dioxide; $\mathrm{PO}_{2}$, partial pressure of oxygen; RVSP, right ventricular systolic pressure; SD, standard deviation.

statistical significance (OR $=1.9608 ; 95 \%$ CI: 1.0007-3.8903; $P=0.0512)$. Patients with elevated NT-pro-BNP levels had a greater hospital LOS (6 days [interquartile range, IQR 5] vs 4 days [IQR 3]; $P=0.0052$ ) compared to patients with normal NT-pro-BNP levels, which was also noted in multivariable model (OR =477.6; 95\% CI: 19-11,993.6; $P<0.001)$. We did not observe any significant differences in the need for NIPPV or MV or in-hospital mortality between the two groups.

\section{Discussion}

Our study is the largest to date to evaluate the role of NTpro-BNP in patients admitted to the hospital for AECOPD while considering the severity of COPD and excluding potential confounders of elevated NT-pro-BNP levels. We found an inverse relationship between elevated NT-proBNP levels and mild left ventricular DD in patients with AECOPD. Patients with elevated NT-pro-BNP levels had lower BMI and albumin levels and elevated BUN levels, were less likely to have grade 1 or grade 2 DD and more likely to have elevated RVSP on echocardiogram. They were

Table 3 Correlation analysis of NT-pro-BNP

\begin{tabular}{lll}
\hline Variables tested & Correlation coefficient & $P$-value \\
\hline NT-pro-BNP and RVSP & $r=0.3658 ; 95 \% \mathrm{Cl}: 0.2060-0.5067$ & $P<0.0001$ \\
NT-pro-BNP and $\mathrm{PCO}_{2}$ & $r=0.2113 ; 95 \% \mathrm{Cl}: 0.0615-0.3519$ & $P=0.006$ \\
NT-pro-BNP and & $r=0.1893 ; 95 \% \mathrm{Cl}: 0.0386-0.3317$ & $P=0.014$ \\
serum bicarbonate & & \\
\hline
\end{tabular}

Abbreviations: BNP, B-type natriuretic peptide; $\mathrm{Cl}$, confidence interval; NT-pro$\mathrm{BNP}, \mathrm{N}$-terminal fragment of pro-BNP; $\mathrm{PCO}_{2}$, partial pressure of carbon dioxide; RVSP, right ventricular systolic pressure. also more likely to be admitted to the ICU and had a greater hospital LOS. NT-pro-BNP levels did not have a significant impact on mortality or the requirement for NIPPV, intubation and MV, or new tracheostomy.

In a previous study, Medina et $\mathrm{al}^{12}$ evaluated the prognostic value of NT-pro-BNP in patients with AECOPD. They excluded patients with HF, depressed ejection fraction, abnormal diastolic function, valvular heart disease, or chronic kidney disease to minimize confounding etiologies for the rise in NT-pro-BNP. They did not, however, limit their study to patients with spirometrically confirmed COPD, and their analysis did not include LOS or the need for NIPPV or MV. They expressed the study outcomes for all chronic pulmonary diseases, which included COPD, chronic asthma, pulmonary fibrosis, and others. Therefore, it is unclear whether the

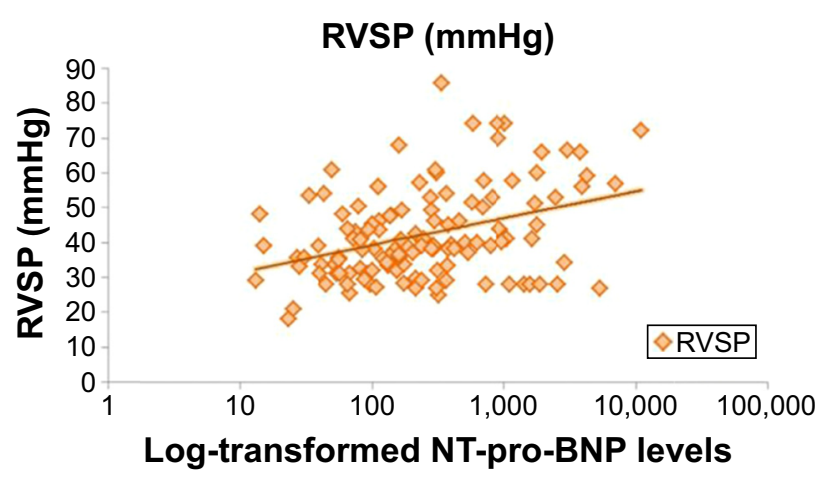

Figure I Scatter plot showing a moderately positive association of NT-pro-BNP levels with echocardiographically estimated RVSP.

Abbreviations: BNP, B-type natriuretic peptide; NT pro-BNP, N-terminal fragment of pro-BNP; RVSP, right ventricular systolic pressure. 
Table 4 Outcomes and interventions according to age-specific NT-pro-BNP levels in patients with AECOPD

\begin{tabular}{|c|c|c|c|c|}
\hline Variables & $\begin{array}{l}\text { All cases } \\
(n=167)\end{array}$ & $\begin{array}{l}\text { Normal NT-pro- } \\
\text { BNP }(n=63)\end{array}$ & $\begin{array}{l}\text { High NT-pro- } \\
\text { BNP }(n=104)\end{array}$ & Significance \\
\hline \multicolumn{5}{|l|}{ Interventions, n (\%) } \\
\hline NIPPV & $83(50)$ & $26(4 I)$ & $57(55)$ & $P=0.1245$ \\
\hline Intubation & $20(12)$ & $7(11)$ & $13(13)$ & $P=0.9824$ \\
\hline Tracheostomy & $4(2)$ & $\mathrm{I}(2)$ & $3(3)$ & $P=0.9925$ \\
\hline \multicolumn{5}{|l|}{ LOS } \\
\hline Number admitted to floor, $\mathrm{n}(\%)$ & $75(45)$ & $36(57)$ & $39(38)$ & $P=0.0207$ \\
\hline Floor median LOS (IQR) & $5(6)$ & $4(3)$ & $6(5)$ & $P=0.0053$ \\
\hline Number admitted to ICU, n (\%) & $92(55)$ & $27(43)$ & $65(63)$ & $P=0.0207$ \\
\hline ICU median LOS (IQR) & $2(2)$ & $2(2)$ & $2(2)$ & $P=0.6868$ \\
\hline Total hospital LOS (IQR) & $5(5)$ & $4(3)$ & $6(5)$ & $P=0.0053$ \\
\hline \multicolumn{5}{|l|}{ Discharge disposition, $\mathrm{n}(\%)$} \\
\hline Home & 149 (89) & $59(94)$ & $90(87)$ & $P=0.2663$ \\
\hline Nursing home & $12(7)$ & $2(3)$ & $10(10)$ & \\
\hline Hospice & $4(2)$ & $2(3)$ & $2(2)$ & \\
\hline In-hospital death & $2(1)$ & $0(0)$ & $2(2)$ & $P=0.5273$ \\
\hline
\end{tabular}

Abbreviations: AECOPD, acute exacerbation of COPD; BNP, B-type natriuretic peptide; ICU, intensive care unit; IQR, interquartile range; LOS, length of stay; NIPPV, noninvasive positive pressure ventilation; NT-pro-BNP, N-terminal fragment of pro-BNP.

outcomes accurately represent a direct association between elevated NT-pro-BNP levels and outcomes in patients with AECOPD. We excluded patients without confirmed COPD by limiting our study to patients with spirometry showing postbronchodilator $\mathrm{FEV}_{1} / \mathrm{FVC}<0.70$, and we further classified the included patients based on the severity of COPD. We did not observe any relationship between elevated NT-pro-BNP levels and COPD severity or home oxygen use at baseline.

The pathophysiological processes leading to elevated NT-pro-BNP levels and the relationships between those processes and adverse outcomes in AECOPD are not well understood. Studies have shown that patients with normal NT-pro-BNP or troponin T levels had lower mortality rates than patients with abnormal levels of those proteins, suggesting possible cardiac involvement. In patients with severe COPD, hypoxia and pulmonary vasoconstriction can lead to pulmonary hypertension and right ventricular dysfunction. Studies of patients with pulmonary hypertension have shown relationships between NT-pro-BNP and invasive pulmonary hemodynamics and echocardiographic measures of right ventricular function. ${ }^{13}$ Another study showed significant correlations between elevated plasma BNP levels and pulmonary artery systolic pressure and left ventricular dysfunction in patients with stable COPD. ${ }^{14}$ High biological variability in NT-pro-BNP levels has been observed among patients with stable chronic HF. ${ }^{15,16}$ It has been unclear whether elevated levels of NT-pro-BNP in patients with COPD are primarily due to left or right heart dysfunction. In our study, we excluded patients with valvular abnormalities or chronic systolic and diastolic HF and included those with grade 1 or grade 2 DD, pulmonary embolism, or atrial fibrillation in a multivariable analysis. Therefore, our findings of elevated echocardiographic RVSP in patients with AECOPD and elevated NT-pro-BNP levels might represent a direct impact of AECOPD on right ventricular function in certain patients. However, we cannot comment on whether the elevated RVSP was associated with an acute insult due to the AECOPD or due to another coexisting condition.

The rate of admission to the ICU for AECOPD ranges between $7 \%$ and $22 \%$ among various studies. ${ }^{17-19}$ In our study, $\sim 55 \%$ of the patients admitted to the hospital with AECOPD were admitted to the ICU. The high rate of admission to the ICU in our study likely reflects local practice, because all patients requiring the new use of NIPPV or MV in our hospital are admitted to the ICU. Patients with AECOPD presenting with elevated NT-pro-BNP levels were more likely to be admitted to the ICU compared with those presenting with normal NT-pro-BNP levels (70\% vs 43\%). The hospital LOS for patients with AECOPD varies with the severity of illness and the requirement for MV. ${ }^{20}$ Nevins et $\mathrm{al}^{21}$ evaluated 60 patients with COPD exacerbation who required $\mathrm{MV}$; the mean duration of $\mathrm{MV}$ was 8.9 days, and the median hospital LOS was 14 days. ${ }^{21}$ In our study, the median LOS for mechanically ventilated patients was 8 days (range: 1-103 days), which is consistent with the study by Nevins et al. ${ }^{21}$ In addition, we reviewed data for patients who were admitted to the hospital but did not require ICU care as well as for patients who required ICU care, which provided us with additional insights. Patients with elevated 
NT-pro-BNP levels had a greater LOS than patients with normal NT-pro-BNP levels whether or not they required ICU admission. Furthermore, patients with elevated NTpro-BNP levels were more likely to be admitted to the ICU, although the overall LOS in the ICU was comparable between the two groups.

Connors et $\mathrm{al}^{22}$ reported an in-hospital mortality rate of $11 \%$ in a cohort of 1,016 patients with hypercapnic respiratory failure secondary to AECOPD. Patients requiring MV had an increased mortality rate. The mortality rate in our study $(1.2 \%)$ was lower than that in the study by Connors et $\mathrm{al}^{22}$ but was more in line with the study by Medina et al, ${ }^{12}$ which showed a 30 -day hospital mortality rate of $1.6 \%$. We attribute those differences to our exclusion criteria, which could have resulted in a younger patient population and excluded patients with other comorbidities such as underlying heart diseases and advanced renal disease that also affect mortality.

In a recent study, Calzetta et $\mathrm{al}^{23}$ explored the teleological activities and paradoxical effects of BNP and its therapeutic implications in chronic obstructive pulmonary disorders. They suggested that BNP is much more than a biomarker as it may modulate the bronchial tone in COPD patients. This effect on bronchial epithelium is independent of its interaction with the parasympathetic system. Broncho-relaxant effect of BNP is thought to be mediated by its interaction with natriuretic peptide receptor A (NPR-A) that is present on the bronchial epithelium. Integrity of the bronchial epithelium is essential for this interaction. Furthermore, BNP has a higher molecular weight compared with currently used bronchodilators, which may lead to technical difficulties in developing a delivery device.

Our study had some limitations. We used a retrospective design and included patients from a single center, which cares for an inner-city population with specific demographic characteristics. Furthermore, our study group was relatively small. Despite finding trends toward greater requirements for NIPPV and MV and a higher mortality rate in patients with elevated NT-pro-BNP levels, those trends were not statistically significant. A larger study might have provided more insight into those important parameters. Echocardiograms were not always performed at the time of obtaining NT-pro-BNP levels, therefore, we cannot definitively exclude that some patients may have developed low ejection fraction or more severe DD in the interim. As the providers were not blinded to patient's laboratory data, we cannot assert if finding a higher NT-pro-BNP level contributed to the decision to admit patients to the ICU. Due to retrospective nature of the study, we cannot accurately assess why the NTpro-BNP was ordered in our study population. To address the potential bias of underlying cardiac disease to the best of our ability, we excluded patients with any of the following characteristics: systolic HF (ejection fraction $<50 \%$ ), valvular heart disease, acute myocardial infarction, and grade 3 or grade 4 DD. We further included patients with grades 1 and 2 DD, atrial fibrillation, pulmonary embolism, and those with elevated serum troponin levels in our analysis and did not find any significant positive association with elevated NT-pro-BNP levels. Our study revealed important associations but could not prove any causation. Despite those limitations, the strengths of our study include the inclusion of carefully selected patients with spirometrically confirmed COPD, the exclusion of confounders of elevated NT-pro-BNP levels including chronic and stable HF, and the inclusion of all patients with AECOPD who were admitted to our hospital, which allowed us to compare the hospital LOS and the requirements for NIPPV and MV between patients with and without elevated NT-pro-BNP levels. To our knowledge, ours is the largest study so far to evaluate the role of NT-pro-BNP in patients admitted to the hospital with AECOPD, including only patients with spirometrically confirmed COPD and excluding potential confounders that can increase NT-pro-BNP levels.

\section{Conclusion}

In our study, patients with AECOPD who had elevated age-specific NT-pro-BNP levels had higher likelihood of ICU admission and longer hospital LOS. Echocardiograms were more likely to show elevated RVSP in patients with elevated NT-pro-BNP levels, suggesting a possible association between COPD and right ventricular dysfunction. Larger prospective studies are needed to validate our findings and to assess whether any direct correlation exists between adverse outcomes of AECOPD and right heart dysfunction.

\section{Author contributions}

All authors contributed toward data analysis, drafting and critically revising the paper, gave final approval of the version to be published, and agree to be accountable for all aspects of the work.

\section{Disclosure}

The authors report no conflicts of interest in this work. 


\section{References}

1. Wheaton AG, Cunningham TJ, Ford ES, Croft JB; Centers for Disease Control and Prevention. Employment and activity limitations among adults with chronic obstructive pulmonary disease - United States, 2013. Morbidity and Mortality Weekly Report (MMWR). 2015;64(11):289-295.

2. Mannino DM, Gagnon RC, Petty TL, Lydick E. Obstructive lung disease and low lung function in adults in the United States: data from the National Health and Nutrition Examination Survey 1988-1994. Arch Intern Med. 2000;160(11):1683-1689.

3. Patel JG, Nagar SP, Dalal AA. Indirect costs in chronic obstructive pulmonary disease: a review of the economic burden on employers and individuals in the United States. Int J Chron Obstruct Pulmon Dis. 2014;9:289-300.

4. Dickstein K, Cohen-Solal A, Filippatos G, et al; ESC Committee for Practice Guidelines (CPG). ESC Guidelines for the diagnosis and treatment of acute and chronic heart failure 2008: the Task Force for the Diagnosis and Treatment of Acute and Chronic Heart Failure 2008 of the European Society of Cardiology. Developed in collaboration with the Heart Failure Association of the ESC (HFA) and endorsed by the European Society of Intensive Care Medicine (ESICM). Eur Heart $J$. 2008;29(19):2388-2442.

5. Doyama K, Fukumoto M, Takemura G, et al. Expression and distribution of brain natriuretic peptide in human right atria. $\mathrm{J} \mathrm{Am} \mathrm{Coll} \mathrm{Cardiol}$. 1998;32(7):1832-1838.

6. Hill NS, Klinger JR, Warburton RR, Pietras L, Wrenn DS. Brain natriuretic peptide: possible role in the modulation of hypoxic pulmonary hypertension. Am J Physiol. 1994;266(3 pt 1):L308-L315.

7. Cargill RI, Lipworth BJ. Atrial natriuretic peptide and brain natriuretic peptide in cor pulmonale. Hemodynamic and endocrine effects. Chest. 1996;110(5):1220-1225.

8. Gale CP, White JE, Hunter A, et al. Predicting mortality and hospital admission in patients with COPD: significance of NT pro-BNP, clinical and echocardiographic assessment. J Cardiovasc Med (Hagerstown). 2011;12(9):613-618

9. Chang CL, Robinson SC, Mills GD, et al. Biochemical markers of cardiac dysfunction predict mortality in acute exacerbations of COPD Thorax. 2011;66(9):764-768.

10. Hoiseth AD, Omland T, Hagve TA, Brekke PH, Soyseth V. NT-proBNP independently predicts long term mortality after acute exacerbation of COPD - a prospective cohort study. Respir Res. 2012;13:97.
11. Buchan A, Bennett R, Coad A, Barnes S, Russell R, Manuel AR. The role of cardiac biomarkers for predicting left ventricular dysfunction and cardiovascular mortality in acute exacerbations of COPD. Open Heart. 2015;2(1):e000052.

12. Medina AM, Marteles MS, Saiz EB, et al. Prognostic utility of NTproBNP in acute exacerbations of chronic pulmonary diseases. Eur $J$ Intern Med. 2011;22(2):167-171.

13. Souza R, Bogossian HB, Humbert M, et al. N-terminal-pro-brain natriuretic peptide as a haemodynamic marker in idiopathic pulmonary arterial hypertension. Eur Respir J. 2005;25(3):509-513.

14. Fijalkowska A, Kurzyna M, Torbicki A, et al. Serum N-terminal brain natriuretic peptide as a prognostic parameter in patients with pulmonary hypertension. Chest. 2006;129(5):1313-1321.

15. Bruins S, Fokkema MR, Romer JW, et al. High intraindividual variation of B-type natriuretic peptide (BNP) and amino-terminal proBNP in patients with stable chronic heart failure. Clin Chem. 2004; 50(11):2052-2058.

16. Inoue $Y$, Kawayama $T$, Iwanaga $T$, Aizawa H. High plasma brain natriuretic peptide levels in stable COPD without pulmonary hypertension or cor pulmonale. Intern Med. 2009;48(7):503-512.

17. Confalonieri M, Garuti G, Cattaruzza MS, et al. A chart of failure risk for noninvasive ventilation in patients with COPD exacerbation. Eur Respir J. 2005;25(2):348-355.

18. Gunen H, Hacievliyagil SS, Kosar F, et al. Factors affecting survival of hospitalised patients with COPD. Eur Respir J. 2005;26(2):234-241.

19. O'Hanlon R, O'Shea P, Ledwidge M, et al. The biologic variability of B-type natriuretic peptide and $\mathrm{N}$-terminal pro-B-type natriuretic peptide in stable heart failure patients. $J$ Card Fail. 2007;13(1):50-55.

20. Groenewegen KH, Schols AM, Wouters EF. Mortality and mortalityrelated factors after hospitalization for acute exacerbation of COPD Chest. 2003;124(2):459-467.

21. Nevins ML, Epstein SK. Predictors of outcome for patients with COPD requiring invasive mechanical ventilation. Chest. 2001;119(6): 1840-1849.

22. Connors AF Jr, Dawson NV, Thomas C, et al. Outcomes following acute exacerbation of severe chronic obstructive lung disease. The SUPPORT investigators (Study to Understand Prognoses and Preferences for Outcomes and Risks of Treatments). Am J Respir Crit Care Med. 1996;154(4 pt 1):959-967.

23. Calzetta L, Orlandi A, Page C, et al. Brain natriuretic peptide: much more than a biomarker. Int J Cardiol. 2016;221:1031-1038.
International Journal of COPD

\section{Publish your work in this journal}

The International Journal of COPD is an international, peer-reviewed journal of therapeutics and pharmacology focusing on concise rapid reporting of clinical studies and reviews in COPD. Special focus is given to the pathophysiological processes underlying the disease, intervention programs, patient focused education, and self management protocols.

\section{Dovepress}

This journal is indexed on PubMed Central, MedLine and CAS. The manuscript management system is completely online and includes a very quick and fair peer-review system, which is all easy to use. Visit http://www.dovepress.com/testimonials.php to read real quotes from published authors. 